\title{
ANALYTICITY PRESERVING PROPERTIES OF RESOLVENTS FOR DEGENERATE DIFFUSION OPERATORS IN ONE DIMENSION
}

\author{
MASAAKI TSUCHIYA
}

\begin{abstract}
Let $L=a(x)\left(d^{2} / d x^{2}\right)+b(x)(d / d x)+c(x)$ be a diffusion operator on a compact interval $I=\left[r_{0}, r_{1}\right]$ investigated by $\mathrm{S}$. N. Ethier. Here, assume that $a, b$ and $c$ are real analytic functions on $I, a(x)>0$ for $x \in\left(r_{0}, r_{1}\right), a\left(r_{i}\right)=$ $0 \leq(-1)^{i} b\left(r_{i}\right)(i=0,1)$, and both $r_{i}(i=0,1)$ are simple zeros of $a(x)$. It is shown that the resolvent $\left\{G_{\lambda}\right\}$ for $L$ has the analyticity preserving property for sufficiently large $\lambda$, so that the equation $(L-\lambda) u=f$ is solvable in the space of real analytic functions on $I$. Some examples are given to show that the condition on $L$ is best possible.
\end{abstract}

1. Introduction. The purpose of this paper is to study the analyticity preserving properties of resolvents for the diffusion operators investigated by Ethier in [1]. This is connected with the solvability of second order linear ordinary differential equations in the space of real analytic functions.

Let $I=\left[r_{0}, r_{1}\right]$ be a compact interval in $(-\infty, \infty)$ and $C^{2}(I)$ be the space of twice continuously differentiable real functions on $I$. Let $L$ be a diffusion operator defined on $C^{2}(I)$ :

$$
L=a(x) \frac{d^{2}}{d x^{2}}+b(x) \frac{d}{d x}+c(x)
$$

where $a, b, c \in C^{\infty}(I), a \geq 0$ and $a\left(r_{i}\right)=0 \leq(-1)^{i} b\left(r_{i}\right)(i=0,1)$. Here, $C^{\infty}(I)$ denotes the space of infinitely differentiable real functions on $I$. Let $C(I)$ be the space of continuous real functions on $I$. Ethier's result contains that an extension of $L$ generates a unique strongly continuous nonnegative semigroup $\left\{T_{t}\right\}$ on $C(I)$ and $T_{t}: C^{\infty}(I) \rightarrow C^{\infty}(I)$ for $t \geq 0$. However, it will be shown that the resolvent $G_{\lambda}=\int_{0}^{\infty} e^{-\lambda t} T_{t} d t$ does not always inherit this property from the semigroup (see Examples 1, 2), so that in such cases $(L-\lambda)\left(C^{\infty}(I)\right) \neq C^{\infty}(I)$. Denote by $C^{\omega}(I)$ the space of real analytic functions on $I$. We will give a best possible condition under which $G_{\lambda}: C^{\omega}(I) \rightarrow C^{\omega}(I)$ for sufficiently large $\lambda$, so that $(L-\lambda)\left(C^{\omega}(I)\right)=C^{\omega}(I)$.

It should be noticed that $L$ does not have the analytic hypoellipticity on $I$, because it has singular points (see Komatsu [3, Theorem 1]).

2. Ethier's result. Our result is based on the definitive result of Ethier on the differentiability preserving properties of the semigroups. We quote part of his result in the form used later. Let $C^{0}(I)=C(I)$ and $C^{n}(I)$ be the space of $n$th continuously differentiable real functions on $I(n=1,2, \ldots)$. For $n=0,1, \ldots, C^{n}(I)$ is the Banach space with the norm \|\|$_{n},\|f\|_{n}=\sum_{k=0}^{n}\left\|f^{(k)}\right\|_{0}$, where \|\|$_{0}$ is the supremum norm.

Received by the editors January 10, 1983.

1980 Mathematics Subject Classification. Primary 34A25, 60J35.

(C) 1984 American Mathematical Society $0002-9939 / 84 \$ 1.00+\$ .25$ per page 
For the coefficients $a, b, c$ of $L$, set

$$
d_{k j}=\left(\begin{array}{c}
k \\
j-2
\end{array}\right) a^{(k-j+2)}+\left(\begin{array}{c}
k \\
j-1
\end{array}\right) b^{(k-j+1)}+\left(\begin{array}{c}
k \\
j
\end{array}\right) c^{(k-j)} \quad(0 \leq j \leq k \leq n),
$$

where $\left(\begin{array}{c}k \\ -2\end{array}\right)=\left(\begin{array}{c}k \\ -1\end{array}\right)=0$.

Ethier's result (see $[1$, Theorem 1]): For $n=1,2, \ldots$,

$$
T_{t}: C^{n}(I) \rightarrow C^{n}(I) \quad(t \geq 0)
$$

and

$$
\left\|T_{t}\right\|_{n} \leq \exp \left(\lambda_{n} t\right) \quad(t \geq 0)
$$

where

$$
\lambda_{n}=\max _{0 \leq j \leq n} \sum_{k=j}^{n}\left\|d_{k j}\right\|_{0}
$$

3. Main result. We set the following assumption on the coefficients of $L$.

Assumption. (1) $a, b, c \in C^{\omega}(I)$,

(2) $a(x)>0$ for $x \in I^{0}=\left(r_{0}, r_{1}\right)$,

(3) $a\left(r_{i}\right)=0 \leq(-1)^{i} b\left(r_{i}\right)(i=0,1)$,

(4) both $r_{i}(i=0,1)$ are simple zeros of $a(x)$.

THEOREM. Under the assumption,

$$
G_{\lambda}: C^{\omega}(I) \rightarrow C^{\omega}(I) \text { for } \lambda>\lambda_{2} .
$$

First, we show the following lemma.

Lemma. Suppose the assumption. If $\lambda \neq c\left(r_{i}\right)(i=0$ or 1$)$, then for any $f \in C^{\omega}(I)$ the equation $(L-\lambda) u=f$ has a real analytic solution $u$ in a neighborhood of $r_{i}(i=0$ or 1).

Proof. Assume that $i=0$. By the assumption on $L$, we can write

$$
a(x)=\left(x-r_{0}\right) \tilde{a}(x),
$$

where $\tilde{a}(x)>0\left(r_{0} \leq x<r_{1}\right)$ and $\tilde{a}(x) \in C^{\omega}(I)$. Therefore, in a small neighborhood of $r_{0}$, the equation $(L-\lambda) u=f$ is equivalent to the equation

$$
\left(x-r_{0}\right) u^{\prime \prime}+\tilde{b}(x) u^{\prime}+\tilde{c}(x) u=\tilde{f}(x),
$$

where $\tilde{b}(x)=b(x) / \tilde{a}(x), \tilde{c}(x)=\{c(x)-\lambda\} / \tilde{a}(x)$ and $\tilde{f}(x)=f(x) / \tilde{a}(x)$. Since $\tilde{c}\left(r_{0}\right) \neq 0$ and $\tilde{b}\left(r_{0}\right) \geq 0$, there is a formal power-series solution of (3.1) at $x=r_{0}$. The formal power-series solution becomes an actual solution of (3.1), because $r_{0}$ is a regular singular point of the operator $\left(x-r_{0}\right)\left(d^{2} / d x^{2}\right)+\tilde{b}(x)(d / d x)+\tilde{c}(x)$ (cf. [2, Theorem 6.1]).

Proof of Theorem. Let $f$ be a real analytic function on $I$. From Ethier's result, it follows that for $\lambda>\lambda_{2}, G_{\lambda} f \in C^{2}(I)$ and $u=G_{\lambda} f$ is a solution of $(L-\lambda) u=-f$ on $I$. Since $a(x)>0$ for all $x \in I^{0}, L-\lambda$ has the analytic hypoellipticity on $I^{0}$ (see [3, Theorem 1]). Therefore, $G_{\lambda} f \in C^{\omega}\left(I^{0}\right)$ for $\lambda>\lambda_{2}$. Suppose that $i=0$. If $\lambda>\lambda_{2}$, then $\lambda \neq c\left(r_{0}\right)$, because $\lambda_{2}>\lambda_{0}$. Therefore, the equation $(L-\lambda) u=-f$ has a real analytic solution $u_{0}$ in a neighborhood of $r_{0}$. 
Hence, for some $\delta>0, u-u_{0}$ is a $C^{2}$-solution of the homogeneous equation

$$
(L-\lambda) v=0
$$

in $\left(r_{0}, r_{0}+\delta\right)$. The equation (3.2) is equivalent to the following equation in $\left(r_{0}, r_{0}+\delta\right)$ :

$$
\left(x-r_{0}\right)^{2} v^{\prime \prime}+\left(x-r_{0}\right) P(x) v^{\prime}+Q(x) v=0,
$$

where $P(x)=\left(x-r_{0}\right) b(x) / a(x), Q(x)=\left(x-r_{0}\right)^{2}\{c(x)-\lambda\} / a(x)$. By the assumption of the theorem, $P\left(r_{0}\right) \geq 0$ and $Q\left(r_{0}\right)=0$. Thus the indicial equation for (3.3) relative to $x=r_{0}$, i.e., $\rho(\rho-1)+P\left(r_{0}\right) \rho+Q\left(r_{0}\right)=0$ has 0 and $1-P\left(r_{0}\right)$ as roots. Therefore, a fundamental system of solutions $\left\{v_{1}(x), v_{2}(x)\right\}$ of (3.3) is given in the following form:

(i) if $\rho_{1} \equiv 1-P\left(r_{0}\right)=0$ or 1 , then

$$
\begin{aligned}
& v_{1}(x)=\left(x-r_{0}\right)^{\rho_{1}} \sum_{n=0}^{\infty} a_{n}\left(x-r_{0}\right)^{n}, \\
& v_{2}(x)=A v_{1}(x) \log \left(x-r_{0}\right)+\sum_{n=0}^{\infty} b_{n}\left(x-r_{0}\right)^{n},
\end{aligned}
$$

where $a_{n}, A, b_{n}$ are real numbers, $a_{0} \neq 0$, and $\sum a_{n}\left(x-r_{0}\right)^{n}$ and $\sum b_{n}\left(x-r_{0}\right)^{n}$ converge in a neighborhood of $r_{0}$;

(ii) if $\rho_{1} \neq 0,1$, then

$$
v_{1}(x)=\left(x-r_{0}\right)^{\rho_{1}} \sum_{n=0}^{\infty} a_{n}\left(x-r_{0}\right)^{n}, \quad v_{2}(x)=\sum_{n=0}^{\infty} c_{n}\left(x-r_{0}\right)^{n},
$$

where $a_{n}$ and $c_{n}$ are real numbers, $a_{0} \neq 0, c_{0} \neq 0$, and $\sum a_{n}\left(x-r_{0}\right)^{n}, \sum c_{n}\left(x-r_{0}\right)^{n}$ converge in a neighborhood of $r_{0}$. Assume that $\delta$ is sufficiently small. Then, there are constants $k_{1}$ and $k_{2}$ such that

$$
u(x)-u_{0}(x)=k_{1} v_{1}(x)+k_{2} v_{2}(x) \quad\left(r_{0}<x<r_{0}+\delta\right) .
$$

In the case (i) with $A=0, u(x)$ is real analytic at $r_{0}$. If $A \neq 0$, then

$$
\begin{aligned}
& k_{2}\left(x-r_{0}\right)^{\rho_{1}} \log \left(x-r_{0}\right) \\
& \quad=\left\{u(x)-u_{0}(x)-k_{1} v_{1}(x)-k_{2} \sum b_{n}\left(x-r_{0}\right)^{n}\right\} / A \sum a_{n}\left(x-r_{0}\right)^{n} .
\end{aligned}
$$

The right side is a $C^{2}$-function on $\left(r_{0}, r_{0}+\delta\right)$, but the left side is not. Therefore, $k_{2}=0$, so that $u(x)=u_{0}(x)+k_{1} v_{1}(x)$ is real analytic at $r_{0}$. In the case (ii) we can prove that $u(x)$ is real analytic at $r_{0}$ in the same way. The function $u$ is also analytic at $r_{1}$. Consequently, $u \in C^{\omega}(I)$.

4. Examples. In this section, it is shown that the consequence of the theorem is not always valid if the condition (2) of the assumption is replaced by the condition $a(x) \geq 0(x \in I)$ or the condition (4) is omitted.

Let us consider a linear ordinary differential equation with analytic data. When this equation has a $C^{\infty}$-solution in an interval, there is a formal power-series solution of the equation at each point of the interval. Further assume that all the singular points of the equation are regular. Then in the same way as the proof of the theorem, it is shown that the $C^{\infty}$-solution is a $C^{\omega}$-solution in the interval.

Let $L$ be a second order linear ordinary differential equation with polynomial coefficients and $u=\sum_{n=0}^{\infty} u_{n}\left(x-x_{0}\right)^{n}$ be a formal solution of $(L-\lambda) u=f$ with 
analytic function $f$. Then the sequence $\left\{n(n-1) u_{n}\right\}$ satisfies a difference equation of Poincaré's type. Hence, the following examples are proved by the fundamental result of Perron in [5].

ExAmPle 1. Let $L=x(x-1)^{2}\left(d^{2} / d x^{2}\right)$ and $I=[0,1]$. Then $G_{\lambda}: C^{\omega}(I) \neq C^{\omega}(I)$ for every $\lambda>0$, so that $G_{\lambda}: C^{\infty}(I) \nrightarrow C^{\infty}(I)$ for every $\lambda>0$.

EXAmple 2. Let $L=x(x-1)^{2}(3-x)\left(d^{2} / d x^{2}\right)$ and $I=[0,3]$. Then the same consequence as in Example 1 is obtained.

EXAMPLE 3. Let $L=x(x-1)(x-2)\left(d^{2} / d x^{2}\right)$. If $I \subset(-\infty, 2)$, then, $(L-\lambda)\left(C^{\omega}(I)\right)=C^{\omega}(I)$ for every $\lambda>0$, and if $0,1,2 \in I$, then $(L-\lambda)\left(C^{\omega}(I)\right) \neq C^{\omega}(I)$ for every $\lambda$.

We prove only Example 1. The others are proved in a similar way. Set $f=$ $(x-1)^{2}$. Suppose that $u=G_{\lambda} f \in C^{\omega}(I)$. Then $(L-\lambda) u=-f$, so that $u$ can be continued analytically into the whole complex plane, because all the singular points of $L-\lambda$ are 0 and 1 . Therefore the radius of convergence of $u(x)=\sum_{n=0}^{\infty} u_{n}(x-1)^{n}$ is infinity. Let $v_{n}=n(n-1) u_{n}$. Then the sequence $\left\{v_{n}\right\}$ satisfies

$$
\begin{gathered}
(1-\lambda / 2) v_{2}=-1, \\
\{1-\lambda / n(n-1)\} v_{n}-v_{n-1}=0 \quad(n \geq 3) .
\end{gathered}
$$

If $\lambda=n(n-1)(n=2,3, \ldots)$, then the sequence $\left\{v_{n}\right\}$ does not exist. This is contradiction. Otherwise, the difference equation (4.2) is of Poincaré's type and its characteristic equation is $z-1=0$. Therefore, from the result of Perron [5, Satz 3] (cf. [4, p. 548]), it follows that there is a fundamental solution $\left\{v_{n}^{\prime}\right\}$ of $(4.2)$ satisfying $\lim \sup _{n \rightarrow \infty} \sqrt[n]{\left|v_{n}^{\prime}\right|}=1$. Since $v_{n} \not \equiv 0, v_{n}=c v_{n}^{\prime}$ for some constant $c \neq 0$. Hence $\lim \sup _{n \rightarrow \infty} \sqrt[n]{\left|u_{n}\right|}=1$. This is contradiction. Consequently, for every $\lambda$, $G_{\lambda} f$ is not real analytic in $I$.

REMARK. Let us consider a second order linear ordinary differential equation with real analytic coefficients. Suppose that the equation has $n(\geq 3)$ singular points (multiple singular points being counted according to their multiplicity) in an interval. Then, from the above examples, it is seen that the equation is not always solvable in the space of real analytic functions on the interval, even if it is locally solvable in this interval.

Acknowledgement. The author is grateful to Mr. Seichi Itatsu for valuable discussion and helpful suggestions, and also to Professor Stewart N. Ethier for permission to quote his result and for valuable suggestions.

\section{REFERENCES}

1. S. N. Ethier, Differentiability preserving properties of Markov semigroups associated with onedimensional diffusions, Z. Wahrsch. Verw. Gebiete 45 (1978), 225-238.

2. M. Hukuhara, Ordinary differential equations, 2nd ed., Iwanami, Tokyo, 1980. (Japanese)

3. H. Komatsu, On the regularity of hyperfunction solutions of linear ordinary differential equations with real analytic coefficients, J. Fac. Sci. Univ. Tokyo Sect. IA Math. 20 (1973), 107-119.

4. L. M. Milne-Thomson, The calculus of finite differences, 2nd. ed., Chelsea, New York, 1981.

5. O. Perron, Über Summengleichungen und Poincarésche Differenzengleichungen, Math. Ann. 84 (1921), 1-15.

Department of Mathematics, College of liberal Arts, Kanazawa University, KANAZAWA, 920 JAPAN 